\title{
Platelet Indices in the Diagnosis of Ventriculoperitoneal Shunt Infection in Children
}

\author{
Umit CELIK ${ }^{1}$, Tamer CELIK ${ }^{2}$, Orkun TOLUNAY ${ }^{3}$, Cigdem DONMEZER ${ }^{3}$, Yurdal GEZERCAN ${ }^{4}$, Kurthan MERT ${ }^{3}$, \\ Ali Ihsan OKTEN ${ }^{4}$
}

${ }^{1}$ Adana Numune Research and Training Hospital, Department of Pediatric Infection, Adana, Turkey

${ }^{2}$ Adana Numune Research and Training Hospital, Department of Pediatric Neurology, Adana, Turkey

${ }^{3}$ Adana Numune Research and Training Hospital, Department of Pediatrics, Adana, Turkey

${ }^{4}$ Adana Numune Research and Training Hospital, Department of Neurosurgery, Adana, Turkey

\section{ABSTRACT}

AIM: Ventriculoperitoneal shunt infection (VPSI) is one of the main causes of mortality and morbidity in hydrocephalus. Platelet indices, mean platelet volume (MPV) and platelet distribution width (PDW) have been evaluated for some infectious diseases. In this study we aimed to investigate whether MPV and PDW values are decisive for the diagnosis of VPSI in children.

MATERIAL and METHODS: Charts of VPSI from 2012 to 2015 were reviewed retrospectively. Twenty-five ventriculoperitoneal shunt infected patients and 25 healthy children as the control group were included in the study. Platelet indices were recorded.

RESULTS: A total of 25 VPSI patients and 25 healthy children were enrolled in the study. Significant differences were detected between the MPV and PDW values in the two groups $(p<0.001)$. ROC curve analysis suggested a cut off point for MPV as below 9.2 $\mathrm{fl}$ (smaller values indicate patients) for the diagnosis of VPSI, with sensitivity, specificity, positive predictive value (PPV), and negative predictive value (NPV) of $80 \%, 92 \%, 91 \%$ and $82 \%$, respectively. For PDW, the cut-off point was 12.25 (larger values indicate pathology) for diagnosis of VPSI, with sensitivity, specificity, PPV and NPV of $68 \%, 80 \%, 77 \%$ and $71 \%$, respectively.

CONCLUSION: Our results suggest that MPV and PDW can be used for the diagnosis of VPSI in children with an accuracy of at least 75\%. Moreover, MPV's specificity is higher than the other platelet parameters and leucocyte count.

KEYWORDS: Ventriculoperitoneal shunt infection, Platelet indices, MPV, PDW

\section{INTRODUCTION}

$\mathrm{T}$ The ventriculoperitoneal shunt has been a main treatment option for hydrocephalus. Ventriculoperitoneal shunt infection (VPSI) is the most common instrumentrelated complication in hydrocephalus patients, being an important cause of mortality and morbidity (7). Early diagnosis and management are important since pediatric patients may present with non-specific symptoms mimicking other infections. As in the initial phase, shunt infection may not appear with neurological symptoms, and it can be difficult to diagnose (3). Besides clinical findings, cerebrospinal fluid (CSF) analysis (white blood cell, glucose and protein concentrations) with cerebrospinal fluid culture positivity is essential for diagnosis, as growing the causative agent in the culture or making a diagnosis by using infection indices is not always easy. Moreover, benefits of white blood cell (WBC) counts, C-reactive protein (CRP) and procalcitonin are limited although these markers support the diagnosis of bacterial infection.

Platelet and platelet indices such as mean platelet volume (MPV) and platelet distribution width (PDW) are parameters that can be easily examined through blood count. Recent years have witnessed the increase in the number of studies that investigate whether platelet indices may play a role in some infectious and inflammatory diseases and may be used as markers $(5,6,8,14,17-19,23)$. 
To our knowledge, there are no studies conducted examining the diagnosis of VPSI using platelet indices. In this study, we aimed to investigate whether MPV and PDW values are decisive for the diagnosis of VPSI in children.

\section{MATERIAL and METHODS}

\section{Patients and Study Design}

The study was approved by the local ethics committee (Approval number: 193). It was conducted in a tertiary training and research hospital. From 2012 to 2015, all patients diagnosed with proven VP shunt infections were evaluated and included in the study. Patients' medical records were examined retrospectively. During the study period, a total of 25 patients with VP shunt infections were included.

Shunt infection diagnosis of the patients was made by a Pediatric Infectious Disease specialist. All of the other possible infection causes were excluded with a detailed history, clinical physical examination and laboratory evaluation.

CSF for analysis (glucose, protein, culture, WBC count with differential, gram stain) was obtained from the ventriculoperitoneal shunt before antibiotic administration. When CSF glucose, protein values and WBC count with differential were consistent with bacterial infection, proper antibiotic treatment was started. When VPSI was diagnosed, the shunt was removed and external drainage was inserted.

Microorganism was grown in the cerebrospinal fluid of each patient.

The microorganisms that were grown in the CSF were as follows: Coagulase negative Staphyloccocus (CoNS) 68\% (17 patients), Pseudomonas aeruginosa 8\% (2 patients), Acinetobacter baumannii in $12 \%$ (3 patients), Klebsiella pneumoniae $8 \%$ (2 patients), and Pseudomonas aeruginosa+Klebsiella pneumoniae 4\% (1 patient).

The control group was selected from healthy children who presented to the outpatient clinic. None of them had infectious or chronic diseases.

The complete blood counts (CBC) had been performed on the first day of admission before antibiotic administration and comprised platelet (PLT) counts, MPV, PDW and WBC counts.

\section{Statistical Analysis}

Statistical analysis was performed using the IBM SPSS Statistics Version 20 statistical software package. Categorical variables were provided as numbers and percentages, numerical variables were given as mean \pm standard deviation. Platelet indices were found to be statistically different between groups, and they were analyzed for diagnostic value in VPSI using Receiver Operating Characteristic (ROC) curve analysis. The areas under the ROC curves (AUC) were calculated and the specificity, sensitivity, positive-predictive value (PPV), negative predictive value (NPV) as well as the accuracy for the platelet, MPV and PDW of the most appropriate cut-off point were calculated for VPSI. $p$ value $<0.05$ was considered statistically significant.

\section{RESULTS}

Twenty-five VSPI patients and 25 healthy children as the control group were included in the study. Within the patients with VPSI, 6 (24\%) were female, $19(76 \%)$ were male and mean age was $11.16 \pm 17.13$ months. In the control group, 14 (54\%) patients were female, 11 male $(46 \%)$ and their mean age was $8.72 \pm 5.42$ months. There was no difference in gender distribution and mean age between the control group and the VPSI patients $(p>0.05)$.

The patient group had a mean MPV of $7.78 \pm 1.59 \mathrm{fl}$, and the control group $10.02 \pm 0.649 \mathrm{fl}$. There were significant differences in the MPV values between the two groups $(p<0.001)$. MPV values were significantly lower than in the control group. The mean PDW values of the patient and control group were $14.558+3.635$ and $11.612 \pm 1.319$, respectively. The PDW values were significantly higher in the VPSI group $(p<0.001)$. The mean platelet counts of the patient and control group were $570.12 \pm 238.48 \mathrm{~mm}^{3}$ and $348.32 \pm 70.551 \mathrm{~mm}^{3}$, respectively. The platelet counts were significantly higher in the VPSI group $(p<0.001)$. The mean white blood cell counts of the patient and control group were $15.67 \pm 7.41 \mathrm{~mm}^{3}$ and $9.43 \pm 2.72 \mathrm{~mm}^{3}$, respectively. The WBC counts were significantly higher in the VPSI group $(p<0.001)$ (Table l).

A ROC curve was plotted to identify the parameters that may be statistically significant in VPSI. For MPV, the calculated area under the curve was $89.8 \%$. When the MPV value was below $9.2 \mathrm{fl}$, sensitivity and specificity were $80 \%$ and $92 \%$, and PPV and NPV were $91 \%$ and $82 \%$ respectively (Table II, Figure 1). For PDW value, the area under the curve was $71 \%$ and cut-off value was higher than 12.25 , sensitivity and specificity were $68 \%$ and $80 \%$, and PPV and NPV were $77 \%$, $71 \%$, respectively (Table II, Figure 2). On the other hand, platelet count was higher than $401.500 \mathrm{~mm}^{3}$, sensitivity and specificity were $80 \%$ and $88 \%$, respectively (Table II, Figure 2). The area under the curve was $77.4 \%$ for WBC count and cut off value was higher than $9.73 \times 10^{3} / \mathrm{UL}$, sensitivity and specificity were $76 \%$ and $64 \%$, and PPV and NPV were $68 \%$ and $73 \%$, respectively (Table II, Figure 2 ).

\section{DISCUSSION}

Shunt infection is a common cause of mortality and morbidity in patients with hydrocephalus and incidence is reported between $5-15 \%$ in literature (16). Especially paediatric patients may apply with diseases mimicking nonspecific findings of other childhood diseases, making early diagnosis vital. Ventriculoperitoneal shunt infection can be seen in functional shunts and may also occur as an important cause of shunt dysfunction. Therefore, early diagnosis and management are important. Although some diagnostic criteria are used, in most cases diagnosis during childhood may not be easy. CSF analysis (protein, glucose, WBC count) provides the warning signs for VPSI. It is often necessary to await CSF culture results.

Platelets play a basic role in hemostasis and thrombosis (21). Platelet indices are parameters that can be easily examined through blood count. Mean platelet volume has been used since the 1970s (11). Platelet indices based on MPV and PDW 
have been emphasized in recent years with respect to some infections and inflammatory conditions $(5,6,14,17-19,23)$. The mean platelet volume shows the average size of platelets and platelet production rate and stimulation (4).

It has been demonstrated in recent years that increased MPV is a risk factor associated with the prognosis of cardiovascular and cerebrovascular diseases $(1,10)$. Moreover, MPV was also evaluated in infectious diseases and it was shown that reduced MPV levels were seen in acute appendicitis, respiratory syncytial virus, and HIV infection in the literature $(5,17,18)$. Within the sepsis studies, there are also different results. Some authors associated poor prognosis with increased MPV levels in sepsis while Bessman and Gardner reported reduced MPV levels in septic patients $(2,20)$. Guclu et al. showed that in patients with severe sepsis, PDW and MPV levels were higher than non-severe sepsis (8). On the other hand, Gunluoglu et al. found lower MPV levels in active pulmonary tuberculosis cases but this did not reflect the severity of disease (9).

Platelets also play an important role in inflammatory response. In the literature, lower MPV levels were correlated with increased disease activity in inflammatory diseases such as ulcerative colitis (23). It is thought that the reason of lower MPV levels observed in inflammatory bowel diseases could be the presence of a defect in the regulation of thrombopoiesis (13).

Platelet indices were also evaluated in central nervous system events. Mean platelet volume and PDW were higher in patients with cerebral venous sinus thrombosis with brain parenchymal lesions and predict the severity of thrombosis (12). Also, mean

Table I: Laboratory Values of Patients with VPSI and Control

\begin{tabular}{lccc}
\hline Characteristics & $\begin{array}{c}\text { Patient group } \\
\mathbf{n}=\mathbf{2 5} \text { mean } \pm \text { SD }\end{array}$ & $\begin{array}{c}\text { Control group } \\
\mathbf{n}=\mathbf{2 5} \text { mean } \mathbf{S D}\end{array}$ & p-value \\
\hline MPV (fl) & $7.78 \pm 1.59$ & $10,02 \pm 0,649$ & $<0.001$ \\
\hline PDW (\%) & $14.558 \pm 3.635$ & $11.612 \pm 1.319$ & $<0.001$ \\
\hline Platelet count $\left(\mathrm{mm}^{3}\right)$ & $570.12 \pm 238.48$ & $348.32 \pm 70.551$ & $<0.001$ \\
\hline White blood cell $\left(\mathrm{mm}^{3}\right)$ & $15.67 \pm 7.41$ & $9.43 \pm 2.72$ & $<0.001$ \\
\hline
\end{tabular}

Table II: Results of ROC Analysis for Markers in Predicting VPSI Compared to Control

\begin{tabular}{lccccccc}
\hline & $\begin{array}{c}\text { Cut-off } \\
\text { point }\end{array}$ & Sensitivity & Specificity & PPV & NPV & AUC & $\begin{array}{c}\text { Asymptomatic 95\% Confidence } \\
\text { Interval Lower/Upper Bound }\end{array}$ \\
\hline MPV (fl) & $<9.2$ & 80 & 92 & 91 & 82 & 0.898 & $0.803 / 0.992$ \\
\hline PDW \% & $>12.25$ & 68 & 80 & 77 & 71 & 0.713 & $0.545 / 0.880$ \\
\hline Platelets $\left(\mathrm{mm}^{3}\right)$ & $>401.5$ & 80 & 88 & 87 & 82 & 0.823 & $0.694 / 0.952$ \\
\hline White blood cell $\left(\mathrm{mm}^{3}\right)$ & $>9.73$ & 76 & 64 & 68 & 73 & 0.774 & $0.642 / 0.907$ \\
\hline
\end{tabular}

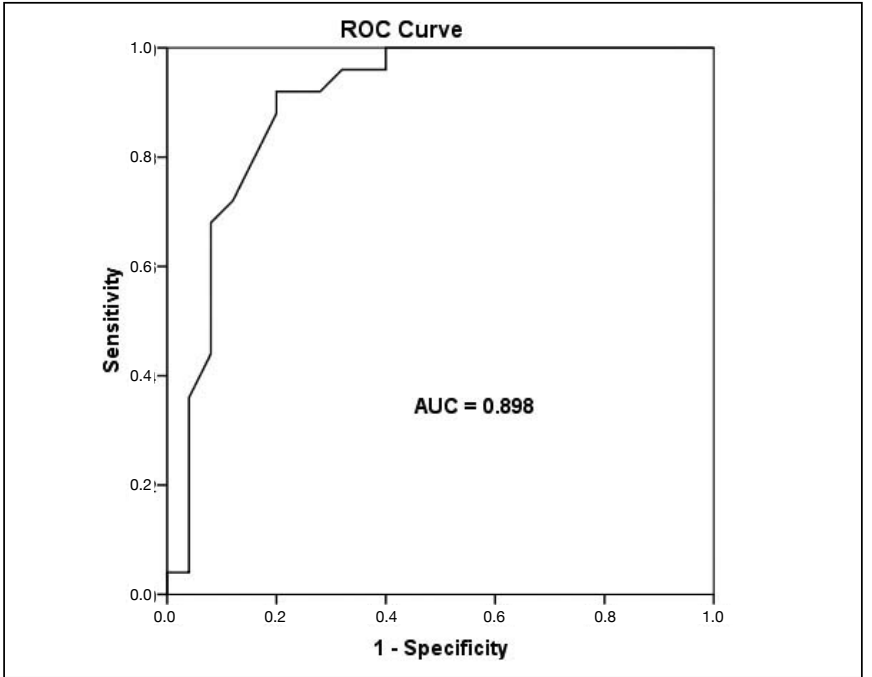

Figure 1: Receiver operating characteristic (ROC) curve for MPV.

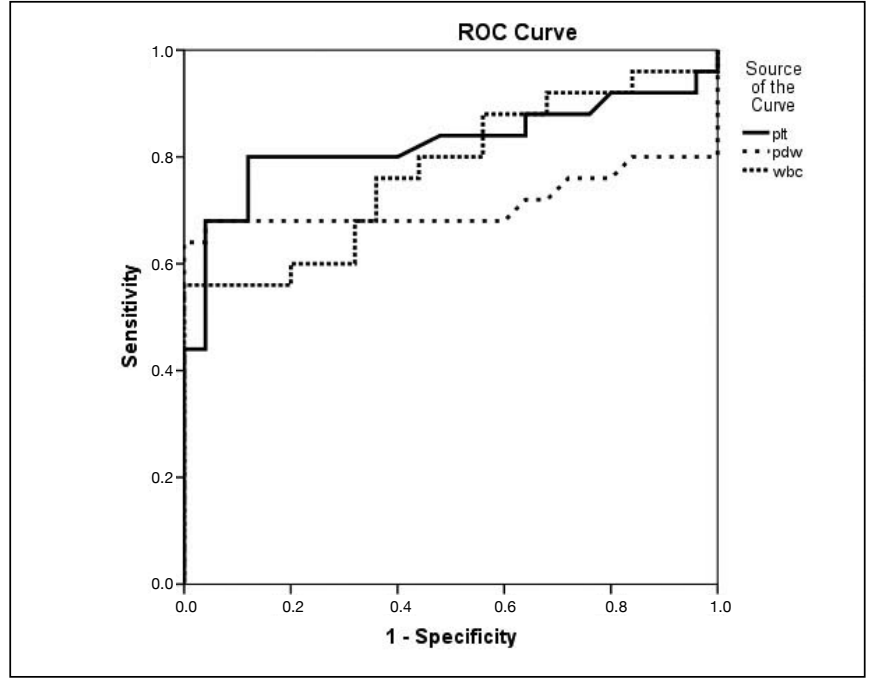

Figure 2: Receiver operating characteristic (ROC) curve for platelet counts, platelet distribution width and white blood cell counts. 
Celik U. et al: Platelet Indices in Ventriculoperitoneal Shunt Infection

platelet volume has been shown to be an independent risk factor for ischemic stroke (15).

Platelet distribution width (PDW), being another platelet index, indicates heterogeneity in platelet sizes. Increased PDW levels indicate increased platelet distribution that is associated with swelling, destruction and immaturity (6). An experimental study suggests that increased PDW levels were associated with endotoxemia (22). Sepsis studies showed that elevated PDW was related to higher mortality outcomes $(6,8)$.

As a result of this study, we saw that platelet indices, especially MPV, are important parameters in patients with VPSI. Mean platelet volume levels were reduced while PDW and platelet levels were increased compared to the control group in patients with VPSI. This study indicated that MPV and PDW values may be used for the diagnosis of VPSI in children with an accuracy of at least $75 \%$. Our findings indicated that MPV levels lower than $9.2 \mathrm{fl}$ have good $(80 \%)$ sensitivity and good (92\%) specificity for VPSI diagnosis. MPV's sensitivity and specificity are higher than the other platelet parameters and leucocyte count. Therefore, MPV may be used as an ancillary test in the diagnosis of VPSI.

\section{CONCLUSION}

To the best of our knowledge, this is the first study to evaluate MPV and PDW in the context of VPSI diagnosis. Mean platelet volume and PDW values would serve as useful parameters for the diagnosis of VPSI that has a high mortality and morbidity rate. We think that clinicians should be aware of this association.

\section{- REFERENCES}

1. Bath P, Algert C, Chapman N, Neal B: Association of mean platelet volume with risk of stroke among 3134 individuals with history of cerebrovascular disease. Stroke 35: 622-626, 2004

2. Bessman JD, Gardner FH: Platelet size in thrombocytopenia due to sepsis. Surg Gynecol Obstet 156:177-180, 1983

3. Conen A, Walti LN, Merlo A, Fluckiger U, Battegay M, Trampuz $A$ : Characteristics and treatment outcome of cerebrospinal fluid shunt-associated infections in adults: A retrospective analysis over an 11-year period. Clin Infect Dis 47:73-82, 2008

4. Ekiz F, Yuksel O, Kocak E, Yilmaz B, Altınbaş A, Coban S, Yuksel I, Uskudar O, Koklu S: Mean platelet volume as a fibrosis marker in patients with chronic hepatitis B. J Clin Lab Anal 25:162-165, 2011

5. Erdem H, Aktimur R, Cetinkunar S, Reyhan E, Gokler C, Irkorucu O, Sozen S: Evaluation of mean platelet volume as a diagnostic biomarker in acute appendicitis. Int J Clin Exp Med 8:1291-1295, 2015

6. Gao Y, Li Y, Yu X, Guo S, Ji X, Sun T, Lan C, Lavergne V, Ghannoum M, Li L: The impact of various platelet indices as prognostic markers of septic shock. PLoS One 9(8):e103761, 2014

7. George R, Leibrock L, Epstein M: Long-term analysis of cerebrospinal fluid shunt infections. A 25-year experience. J Neurosurg 51: 804-811, 1979
8. Guclu E, Durmaz Y, Karabay O: Effect of severe sepsis on platelet count and their indices. Afr Health Sci 13:333-338, 2013

9. Gunluoğlu G, Yazar EE, Veske NS, Seyhan EC, Altin S: Mean platelet volume as an inflammation marker in active pulmonary tuberculosis. Multidiscip Respir Med 28:11, 2014

10. Huczek Z, Kochman J, Filipiak KJ, Horszczaruk GJ, Grabowsk M, Piatkowski R, Wilczynska J, Zielinski A, Meier B, Opolski G: Mean platelet volume on admission predicts impaired reperfusion and long-term mortality in acute myocardial infarction treated with primary percutaneous coronary intervention. J Am Coll Cardiol 46:284-290, 2005

11. Jackson SR, Carter JM: Platelet volume: Laboratory measurement and clinical application. Blood Rev 7:104-113, 1993

12. Kamisli O, Kamisli S, Kablan Y, Gonullu S, Ozcan C: The prognostic value of an increased mean platelet volume and platelet distribution width in the early phase of cerebral venous sinus thrombosis. Clin Appl Thromb Hemost 19(1):2932, 2013

13. Kapsoritakis AN, Koukourakis MI, Sfiridaki A, Potamianos SP, Kosmadaki MG, Koutroubakis IE, Kouroumalis EA: Mean platelet volume: A useful marker of inflammatory bowel disease activity. Am J Gastroenterol 96(3):776-781, 2001

14. Karadag-Oncel E, Ozsurekci Y, Kara A, Karahan S, Cengiz $A B$, Ceyhan $M$ : The value of mean platelet volume in the determination of community acquired pneumonia in children. Ital J Pediatr 39:16, 2013

15. Mayda-Domaç F, Misirli H, Yilmaz M: Prognostic role of mean platelet volume and platelet count in ischemic and hemorrhagic stroke. J Stroke Cerebrovasc Dis 19(1):66-72, 2010

16. Piatt JH, Carlson CV: A search for determinants of cerebrospinal fluid shunt survival: A retrospective analysis of a 14 year institutional experience. Pediatr Neurosurg 19:233241, 1993

17. Qadri S, Holman S, Dehovitz J, Crystal H, Minkoff H, Lazar JM: Mean platelet volume is decreased in HIV-infected women. HIV Med 14(9):549-555, 2013

18. Renshaw AA, Drago B, Toraya N, Gould EW: Respiratory syncytial virus infection is strongly correlated with decreased mean platelet volume. Int J Infect Dis 17(9):678-680, 2013

19. Sayit AT, Gunbey PH, Terzi Y: Is the mean platelet volume in patients with acute cholecystitis an inflammatory marker? J Clin Diagn Res 9(6):TC05-7, 2015

20. Van der Lelie J, Von dem Borne AK: Increased mean platelet volume in septicaemia. J Clin Pathol 36:693-696, 1983

21. Weyrich AS, Lindemann S, Zimmerman GA: The evolving role of platelets in inflammation. J Thromb Haemost 1(9):18971905, 2003

22. Yilmaz Z, Erap O, Ilcol YO: Evaluation of platelet count, and its association with plateletcrit, mean platelet volume, and platelet size distribution width in a canine model of endotoxemia. Vet Clin Pathol 37:159-163, 2008

23. Yüksel O, Helvaci K, Başar O, Köklü $S$, Caner $S$, Helvaci N, Abayli E, Altiparmak E: An overlooked indicator of disease activity in ulcerative colitis: Mean platelet volume. Platelets 20(4):277-281, 2009 